\title{
Les bétons autoplaçants renforcés par un fibrage mixte et multi-échelle : de la formulation au comportement mécanique
}

\author{
H. Oucief ${ }^{1,}$, A. Turatsinze ${ }^{2}$, K. Fanutza ${ }^{2}$ et M. F. Habita \\ 'Département de génie civil, Université Badji-Mokhtar, Annaba (Algérie) \\ ${ }^{2}$ Laboratoire de matériaux et durabilité des constructions(LMDC), INSA de Toulouse \\ (France)
}

(Reçu le 07 Décembre 2005, accepté le 15 Avril 2006)

*Correspondance,courriel : oucief@yahoo.fr

\section{Résumé}

Dans la plupart des cas, les bétons autoplaçants (BAP) renforcés de fibres, contiennent seulement un type de fibre. L'utilisation de deux types de fibres ou plus avec des dimensions différentes et des dosages appropriés peut potentiellement non seulement améliorer les propriétés intrinsèques du béton autoplaçant, mais peut aussi contribuer à un gain conséquent de fibres. La combinaison des fibres qui est souvent appelée hybridation ou mixage est étudiée dans cet article pour des bétons autoplaçants. Des mélanges mixtes ou hybrides ont été formulés avec des fibres d'acier et de polypropylène de différentes longueurs et dosage. Des essais de ténacité en flexion ont été réalisés et les résultats ont été intensivement analysés afin d'évaluer la synergie optimale pour chaque mélange. Se basant sur divers arrangements (combinaisons), l'étude met en évidence l'aspect quantitatif des fibres pour améliorer les performances du composite fibré.

Mots-clés : Bétons, autoplaçants, fibrage, mixte, multi-échelle, ténacité.

\section{Abstract}

\section{Hybrid fiber reinforced self-compacting concrete from formulation to mechanical behavior}

In most cases, fiber reinforced self-compacting concrete (FRSCC) contains only one type of fiber. The use of two or more types of fibers in a suitable combination may potentially not only improve the overall properties of self-compacting concrete, but may also result 
in performance synergy. The combining of fibers, often called hybridization, is investigated in this paper for a cimentous matrix. Control, single, two fibers hybrid composites were cast using different fiber type steel and polypropylene with different sizes. Flexural toughness tests were performed and results were extensively analyzed to identify synergy, if any, associated with various fiber combinations. Based on various analysis schemes, the paper identifies fiber combinations that demonstrate maximum synergy in terms of flexural toughness.

Keywords : Concrete, self-compacting, fibers, hybrid, synergy.

\section{Introduction}

En Europe et en France en particulier, l'intérêt pour ces bétons est apparu seulement vers la fin des années 90 aussi bien dans le domaine des bétons prêts à l'emploi (BPE) que dans celui de la préfabrication.

Ces bétons très fluides, mis en place sans vibration même dans des structures complexes et fortement ferraillés. Toutefois comme pour tout nouveau matériau, il est nécessaire de connaître les spécificités des bétons autoplaçants (BAP) (ou des BAN : bétons autonivelants utilisés pour les dallages) [1].

Les BAP sont caractérisés par une fluidité importante, une diminution du bruit de la pénibilité du travail, l'augmentation des cadences de coulage et surtout une diminution de la ségrégation et du ressuage (par rapport au béton fibré).

Pour obtenir ces propriétés et pour un meilleur arrangement granulaire on ajoute de fortes teneurs en addition minérales: fumées de silice, laitiers, addition calcaire, cendres volantes, (Environ $200 \mathrm{Kg} / \mathrm{m}^{3}$ de filler calcaire pour $300 \mathrm{Kg} / \mathrm{m}^{3}$ de ciment). Cependant, une formulation préalable doit être mise en place afin d'optimiser la composition du BAP renforcé par des fibres d'acier et polymériques afin d'assurer une meilleure ouvrabilité (maniabilité) de ces derniers.

Une étude qui contribue à la compréhension des effets des paramètres de mélange, tels que le volume et la longueur des fibres, le volume des granulats et la composition du béton, ainsi que l'évaluation de l'influence de ces paramètres sur les caractéristiques du béton frais (rhéologie, capacité de remplissage et stabilité) et durci (tenacité, résistance en traction et en compression) semble être très utile [2].

Il a été démontré que pour une même teneur et un même type de fibres, l'efficacité des fibres de $30 \mathrm{~mm}$ est meilleur que celle de $45 \mathrm{~mm}$ aussi bien pour les résistances à la flexion que pour les déformations post-pic [3]. Ce comportement est expliqué par les aspects de porosité, d'ouvrabilité et de mise en œuvre, qui sont plus favorables pour le cas des fibres de $30 \mathrm{~mm}$. De plus il apparaît qu'une fibre d'acier de section plus fine 
permet une meilleure liaison d'interface fibre-matrice. Dans ce cas une teneur de 0,6\% en fibres a la même efficacité que l'utilisation de $2 \%$ de fibres plus grosses et ce pour un même béton [3].

Des associations de fibres de différentes caractéristiques commencent à être utilisées. Banthia et al. [4] montrent que l'association des fibres synthétiques et des fibres métalliques peut donner aux BAP des comportements plus ductiles, que dans le cas des bétons fibrés seulement avec les fibres métalliques. Kawamata et al. [5] ont essayé d'utiliser dans le cas des bétons autoplaçants des fibres longues en association avec les fibres courtes. Selon ces auteurs, les fibres courtes empêchent la formation des petites fissures, retardant ainsi la formation des macro fissures, qui seront à leur tour reprises par les fibres les plus longues et demandent une plus grande dissipation d'énergie pour rompre. Par là la ductilité du matériau serait augmentée.

Cependant, les fibres étant très chères sur le plan économique, la recherche d'une relation entre le dosage et nombre de fibres peut constituer une solution pour les fabricants. Ainsi la synergie réside-t-elle dans les nombres?

Le présent travail traite d'un mélange de fibres de différentes dimensions et propriétés des fibres métalliques de fonte amorphe adhérente $(20 \mathrm{~mm}$ et $30 \mathrm{~mm})$ et des fibres synthétiques glissantes ( $40 \mathrm{~mm}$ et $50 \mathrm{~mm}$ ).

Le but de cette étude est l'optimisation du dosage en fibres en fonction des longueurs, et la détermination de la ténacité des matériaux optimisés en fonction des énergies dépensées au cours de la rupture sous chargement. Cette ténacité pourra être quantifiée en mesurant l'aire sous la courbe force-déplacement.

Cette approche se basera sur le comportement du matériau bi-fibré en tenant compte du nombre de fibres incorporées connaissant le dosage et la longueur de ces dernières. II est évident que le nombre de fibres pourra être déterminé connaissant le dosage, la densité et les caractéristiques géométriques des fibres.

La démarche visera à réduire les coûts plus ou moins élevés des fibres; en d'autres termes, optimiser le dosage quantitatif $\left(\mathrm{kg} / \mathrm{m}^{3}\right)$ des fibres en tenant compte des facteurs économiques: atteindre les résistances souhaitées avec un moindre dosage (moindre coût).

\section{Choix de base}

\section{2-1. Fibres utilisées}

Les fibres utilisées sont des fibres (rubans) métalliques amorphe à base de fonte de longueurs respectives 20 et $30 \mathrm{~mm}$. L'idée de base étant d'utiliser la fonte préfabriquée, 
de la modifier pour la rendre amorphisable dans le but d'obtenir un matériau à propriétés mécaniques et chimiques intéressantes, à savoir :

- La bonne résistance aux agents agressifs et leur haut degré d'inoxydabilité.

- L'absence de problème de malaxage, contrairement à certaines autres fibres.

- La bonne adaptation au procédé de projection par voie sèche.

L'adhérence fibre-béton est généralement faible du fait qu'il n'y a aucune liaison chimique possible entre les fibres et la matrice. Par contre il faut noter l'excellente résistance de ces fibres vis-à-vis des acides, des sels, et des alcalins de béton. Leurs longueurs sont respectivement de 40 et $50 \mathrm{~mm}$. Ces deux types de fibres peuvent être utilisées sans aucune réserve dans les matrices cimenteuses. Les fibres en polypropylène dites aussi synthétiques glissantes et à faible module d'élasticité peuvent être utilisées pour remplacer une partie des fibres métalliques.

En respect des dosages pratiqués dans les éléments de structure, la teneur en fibres au mètre cube de béton est fixé à :

- $20 \mathrm{Kg} / \mathrm{m}^{3}$ Iorsqu' on utilise des fibres métalliques (FM) seules.

- $9 \mathrm{Kg} / \mathrm{m}^{3}$ lorsqu'on utilise des fibres synthétiques (FS) seuls.

- (10 FM +4,5 FS) Kg/m $\mathrm{m}^{3}$ lors de l'emploi simultané des fibres métalliques et synthétiques.

Ces teneurs diffèrent selon la nature des fibres. En effet elles donnent un rapport entre le nombre de fibres métalliques et celui des fibres synthétiques (par $\mathrm{m}^{3}$ ) de béton qui est de l'ordre de grandeur du rapport des densités.

Les propriétés des fibres utilisées sont mentionnées dans le Tableau 1.

Tableau 1 : propriétés des fibres utilisées

\begin{tabular}{|c|c|c|c|c|}
\hline Type de fibre & \multicolumn{2}{|c|}{$\begin{array}{l}\text { Métallique amorphe } \\
\text { SEVA }\end{array}$} & \multicolumn{2}{|c|}{$\begin{array}{l}\text { Polypropylène } \\
\text { GRACE }\end{array}$} \\
\hline Longueur & $20 \mathrm{~mm}$ & $30 \mathrm{~mm}$ & $40 \mathrm{~mm}$ & $50 \mathrm{~mm}$ \\
\hline Section transversale & \multicolumn{2}{|c|}{ Rectangulaire $1,6 * 0,03 \mathrm{~mm}$} & \multicolumn{2}{|c|}{$\begin{array}{l}\text { Rectangulaire } \\
1,6 * 0,4 \mathrm{~mm}\end{array}$} \\
\hline Densité & \multicolumn{2}{|c|}{7,2} & \multicolumn{2}{|c|}{0,92} \\
\hline Résistance à la traction & \multicolumn{2}{|c|}{$2000 \mathrm{Mpa}$} & \multicolumn{2}{|c|}{$310 \mathrm{Mpa}$} \\
\hline Module d'élasticité & \multicolumn{2}{|c|}{ 140Gpa } & \multicolumn{2}{|c|}{$4,3 \mathrm{Gpa}$} \\
\hline $\begin{array}{l}\text { Résistance aux:sels, } \\
\text { acides, alcalins }\end{array}$ & \multicolumn{2}{|c|}{ Inoxydable } & \multicolumn{2}{|c|}{ Haute } \\
\hline
\end{tabular}




\section{2-2. Optimisation des compositions des bétons autoplaçants bi-fibrés}

L'objectif prioritaire de cette approche, est l'étude du comportement mécanique d'un béton autoplaçant bi-fibré et à matrice cimenteuse constante.

Influence de la longueur de la fibre sur la capacité portante maximale $F_{\max }$.

Etude de l'influence du rapport dosage/longueur (nombre de fibres contenues dans la matrice cimenteuse) sur le comportement du matériau.

Et enfin optimisation du dosage.

Pour ce faire nous avons adopté une formulation prédéfinie, tout en respectant la réalité économique, pour le choix des fines (ciment et addition) et des granulats les plus proches possibles du lieu de confection [6].

Le superplastifiant utilisé est un de ceux les plus utilisés pour la confection des bétons autoplaçants : son mode d'action est à la fois électrostatique et stérique.

Les constituants sont regroupés dans le Tableau 2.

Tableau 2 : Composants utilisés pour la fabrication des bétons autoplaçants.

\begin{tabular}{|c|c|c|}
\hline Matériau & Origine & $\begin{array}{c}\text { Masse Volumique en } \\
\mathbf{K g} / \mathbf{m}^{3}\end{array}$ \\
\hline Ciment & CEM I 52,5 (Lafarge, Martres) & 3100 \\
\hline Addition & $\begin{array}{c}\text { Filler calcaire (classe M, la } \\
\text { provençale) }\end{array}$ & 2700 \\
\hline Sable & $\begin{array}{c}0 / 4 \mathrm{~mm} \text { roulé alluvionnaire } \\
\text { (Garone) }\end{array}$ & 2620 \\
\hline Gravier & $\begin{array}{c}4 / 10 \mathrm{~mm} \text { roulé alluvionnaire } \\
\text { (Garone) }\end{array}$ & 1115 \\
\hline Superplastifiant & $\begin{array}{c}\text { SIKA 3030 à base de copolymère } \\
\text { acrylique }\end{array}$ & \\
\hline
\end{tabular}

Sept types de bétons ont été retenus. Les paramètres d'optimisation sont : le dosage et les dimensions des fibres.

Les abréviations suivantes sont utilisées :

BAP : Béton autoplaçant témoin sans fibres

FM: fibres métalliques

FS: fibres synthétiques

FMS : fibres métalliques et synthétiques.

BAPFMS: béton autoplaçant avec fibres métalliques et synthétiques.

BAPFMS1: $\left[10 \mathrm{Kg} / \mathrm{m}^{3} \mathrm{FM}(\mathrm{I}=30 \mathrm{~mm})+4,5 \mathrm{Kg} / \mathrm{m}^{3} \mathrm{FS}(\mathrm{I}=50 \mathrm{~mm})\right]$

BAPFMS2: $\left[10 \mathrm{Kg} / \mathrm{m}^{3} \mathrm{FM}(\mathrm{l}=20 \mathrm{~mm})+4,5 \mathrm{Kg} / \mathrm{m}^{3} \mathrm{FS}(\mathrm{l}=50 \mathrm{~mm})\right]$ 
BAPFMS3: [6, $\left.75 \mathrm{Kg} / \mathrm{m}^{3} \mathrm{FM}(\mathrm{I}=20 \mathrm{~mm})+4,5 \mathrm{Kg} / \mathrm{m}^{3} \mathrm{FS}(\mathrm{I}=50 \mathrm{~mm})\right]$

BAPFMS4: $\left[5 \mathrm{Kg} / \mathrm{m}^{3} \quad F M(I=20 \mathrm{~mm})+4,5 \mathrm{Kg} / \mathrm{m}^{3} \mathrm{FS}(I=50 \mathrm{~mm})\right]$

BAPFMS5: $\left[6,75 \mathrm{Kg} / \mathrm{m}^{3} \mathrm{FM}(\mathrm{l}=20 \mathrm{~mm})+3,6 \mathrm{Kg} / \mathrm{m}^{3} \mathrm{FS}(\mathrm{l}=40 \mathrm{~mm})\right]$

BAPFMS6: $\left[6,75 \mathrm{Kg} / \mathrm{m}^{3} \mathrm{FM}(\mathrm{I}=20 \mathrm{~mm})+4,5 \mathrm{Kg} / \mathrm{m}^{3} \mathrm{FS}(\mathrm{I}=40 \mathrm{~mm})\right]$

Comme indiqué précédemment la composition optimale retenue est donnée à $10 \mathrm{Kg} / \mathrm{m}^{3}$ de fibres métalliques, soit la moitié de la teneur de base et le complément en fibres synthétiques soit de $4,5 \mathrm{Kg} / \mathrm{m}^{3}$, ce qui donne un mixage compatible vis-à-vis du rôle de chaque type de fibres. La composition retenue est la suivante (Tableau 3).

Tableau 3: composition des bétons autoplaçants bi-fibrés étudiés

\begin{tabular}{|c|c|}
\hline BAPFMS & $\mathbf{K g} / \mathbf{m}^{3}$ de béton \\
\hline Ciment C & 340 \\
\hline Filler calcaire F & 213 \\
\hline Sable S & 884 \\
\hline Gravier G & 553 \\
\hline Superplastifiant SP (sika 3030) & 4,08 \\
\hline Fibres Métalliques FM & Variable en dosage et en longueur \\
\hline Fibres Synthétiques FS & Variable en dosage et en longueur \\
\hline Eau Totale & 210 \\
\hline
\end{tabular}

\section{2-3. Fabrication des bétons - Séquence de malaxage - Conservation des éprouvettes}

Incorporation des gros gravillons, du ciment, du filler ef du sable et malaxage durant 30 secondes.

Ajout des fibres et malaxage pour 60 secondes.

Ajout de l'eau d'apport et du superplastifiant dilué dans l'eau, et malaxage pendant 210 secondes. La durée effective totale du malaxage est de 05 minutes.

Après la réalisation des essais d'ouvrabilité, les bétons ont été remalaxés pendant 01 minute puis déchargés dans une brouette pour leur mise en place dans les moules. Les moules remplis (sans aucune vibration) ont été immédiatement couverts, placées à $20^{\circ} \mathrm{C}$, puis les éprouvettes ont été démoulées $24 \mathrm{H}$ après le coulage. Et enfin, elles ont été conservées dans une salle humide jusqu'aux échéances d'essais mécaniques. 


\section{2-4. Processus expérimental}

Les éprouvettes en béton auto-plaçant bi-fibrés ont été confectionnées et préparées auparavant afin d'évaluer les résistances mécaniques en compression simple, et en flexion 04 points comme le montre le schéma de la Figure 1.

Les éprouvettes pour les essais en compression simple sont cylindriques de $110 \mathrm{~mm}$ de diamètre et $220 \mathrm{~mm}$ de hauteur et celles pour les essais en flexion sont prismatiques de section $100 * 100 * 500 \mathrm{~mm}$.

Une série de 03 éprouvettes a été utilisée pour chaque type d'essai. La date d'échéance des essais a été établie à 7 jours après le coulage pour les essais en compression et à 8 jours pour les essais en flexion 4 points.

En flexion et dans le but d'imposer la formation d'une fissure unique dans la région intérieur centrale de la poutre, une entaille de $10 \mathrm{~mm}$ de profondeur a été prévue par sciage à mi-portée des éprouvettes.

Les essais de flexion ont été effectués sur une machine de capacité $50 \mathrm{Kn}$, et grâce à une chaîne d'acquisition de données, les paramètres d'asservissement sont enregistrés et les informations relatives à l'essai stockées sur des disquettes, qui seront ultérieurement traitées et exploitées.

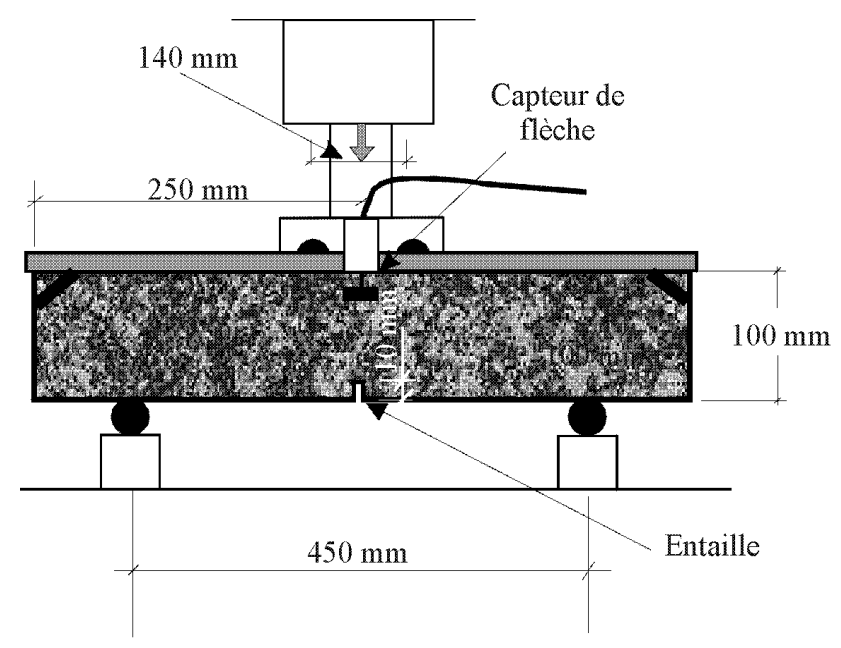

Figure 1 : schéma du dispositif d'essai de flexion 4 points

L'essai asservi en déplacement, a été contrôlé par la valeur de la flèche incrémentée de $0,05 \mathrm{~mm} / \mathrm{mn}$ pour les déformations jusqu'à $0,4 \mathrm{~mm}$, puis à $0,1 \mathrm{~mm} / \mathrm{mn}$ jusqu'à $1,0 \mathrm{~mm}$ et ensuite à $0,5 \mathrm{~mm} / \mathrm{mn}$ jusqu'à la fin de l'essai. La mesure de la flèche a été suivie jusqu'à $6,5 \mathrm{~mm}$.

Les courbes If force-flèche ॥ obtenues permettent de caractériser la réponse des 
composites optimisés par la charge maximale $\mathrm{F}_{\max }$ ainsi que par la flèche maximale $\delta_{\max }$ associée à cette charge maximale: cette force correspond à la capacité maximale de déformation avant la localisation de la microfissure.

\section{Résultats et discussion}

\section{3-1. Indice de ténacité en flexion}

La ténacité des matériaux peut-être évaluée à partir de l'énergie dépensée au cours de leur rupture sous chargement. On peut la quantifier en mesurant l'aire sous la courbe force déplacement. Grâce à l'augmentation de la résistance post-pic on peut constater l'évolution de l'énergie dépensée lors de la rupture. On peut aussi caractériser le comportement élasto-plastique des matériaux au travers des indices et relation de ténacité.

Selon la norme ASTM C108 [6], on évalue l'aire située sous la courbe effort-déformation pour des valeurs particulières de la déformation. 0 n relève, de la même manière, l'aire mesurée jusqu'à la valeur de déflexion " $\delta$ " entraînant la première fissure. Les rapports entre les valeurs des énergies représentées par ces points particuliers et l'énergie consommée jusqu'au point de première fissure, et ainsi, une valeur d'un indice de ténacité " $I_{x}$ " est obtenue. Les valeurs qui sont préconisées par la norme ASTM C 108 sont: $3 \delta$ pour $\mathrm{I}_{5 ;} 5.5 \delta$ pour $\mathrm{I}_{10}$ et $15.5 \delta$ pour $\mathrm{I}_{30}$.

3-2. Influence du nombre et les dimensions des fibres métalliques sur le composite bi-fibré

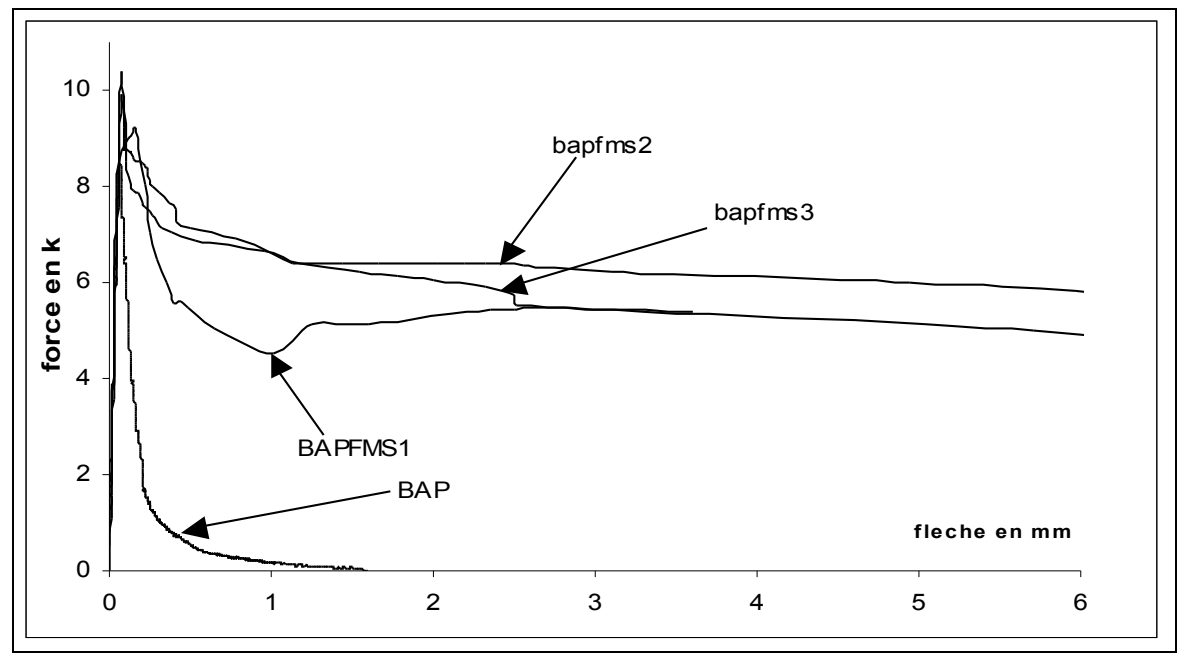

Figure 2 : Courbes effort-flèche : comparaison entre bapfmsl, bapfms2 et bapfms3 
BAPFMS1 et BAPFMS2: [même dosage en fibres polypropylène (pp), même dosage en fibres métalliques mais nombre de fibres $(I=20 \mathrm{~mm})$ dans BAPFMS2 est supérieur au nombre de fibres $(I=30 \mathrm{~mm})$ dans BAPFMSI]

II apparaît clairement d'après les courbes de la Figure 2 qu'un gain de résistance est enregistré au niveau des BAPFMS2. Ce gain est traduit par une augmentation de la capacité portante. En effet pour une même teneur en fibres (dosage égal), l'efficacité des fibres de $20 \mathrm{~mm}$ est meilleur que celles des fibres de $30 \mathrm{~mm}$, aussi bien pour les résistances en flexion que pour l'énergie de rupture. Cet aspect est lié directement à certains aspects rhéologiques, tel que la porosité, l'ouvrabilité, et la mise en œuvre qui sont plus favorables pour les fibres de $20 \mathrm{~mm}$ (résistance d'autant plus forte que les fibres sont en plus grande quantité).

BAPFMS1 et BAPFMS3 : [même dosage en fibres PP, dosage différent en fibres métalliques avec nombre de fibres $(I=20 \mathrm{~mm})$ dans BAPFMS3 égal au nombre de fibres $(I=30 \mathrm{~mm})$ dans BAPFMSI]

La capacité portante pour les BAPFMS3 a considérablement augmenté et la différence est significative en comparaison avec les BAPFMSI comme le montrent les courbes correspondantes de la Figure 2.

Les fibres métalliques de longueurs $I=20 \mathrm{~mm}$ et $\mathrm{I}=30 \mathrm{~mm}$ ont leur domaine d'efficacité à des niveaux de flèche différents, les résultats sont visualisés selon deux échelles. La première pour les flèches jusqu'à $1,00 \mathrm{~mm}$, et la deuxième pour les flèches jusqu'à 5,00 $\mathrm{mm}$.

Cas de flèches jusqu'à $1,00 \mathrm{~mm}:$ on constate que les valeurs des résistances en flexion, les pentes de la zone de comportement linéaire et les comportements post-pic sont fortement influencés par l'élancement des fibres.

On peut observer d'après les courbes de la Figure 3 que par rapport au BAPFMSI, les fibres courtes améliorent la résistance. Le nouveau pic de résistance ainsi obtenu est plus arrondi et maintient une résistance élevée jusqu'à des valeurs de flèches plus importantes. Les énergies de rupture dissipées sont sensiblement égales.

La Figure 2 montre la tendance de comportement aux fortes flèches, jusqu'à $5,00 \mathrm{~mm}$ associées à de grandes ouvertures. Dans ce domaine de flèches ou d'ouvertures de fissures, alors que la résistance apportée par les fibres métalliques adhérentes devient quasiment nulle pour les deux types de mélanges, les fibres glissantes affichent leur meilleure performance. Le comportement intermédiaire du fibrage mixte, cumulant des avantages des deux types de fibres, est confirmé jusqu'aux plus grandes flèches ou ouvertures de fissures.

BAPFMS1 et BAPFMS4: [même dosage en fibres Polypropylène pp, différent dosage en fibres métalliques avec nombre de fibres $(I=20 \mathrm{~mm})$ dans BAPFMS4 inférieur aux nombre de fibres $(I=30 \mathrm{~mm})$ dans BAPFMSI] 


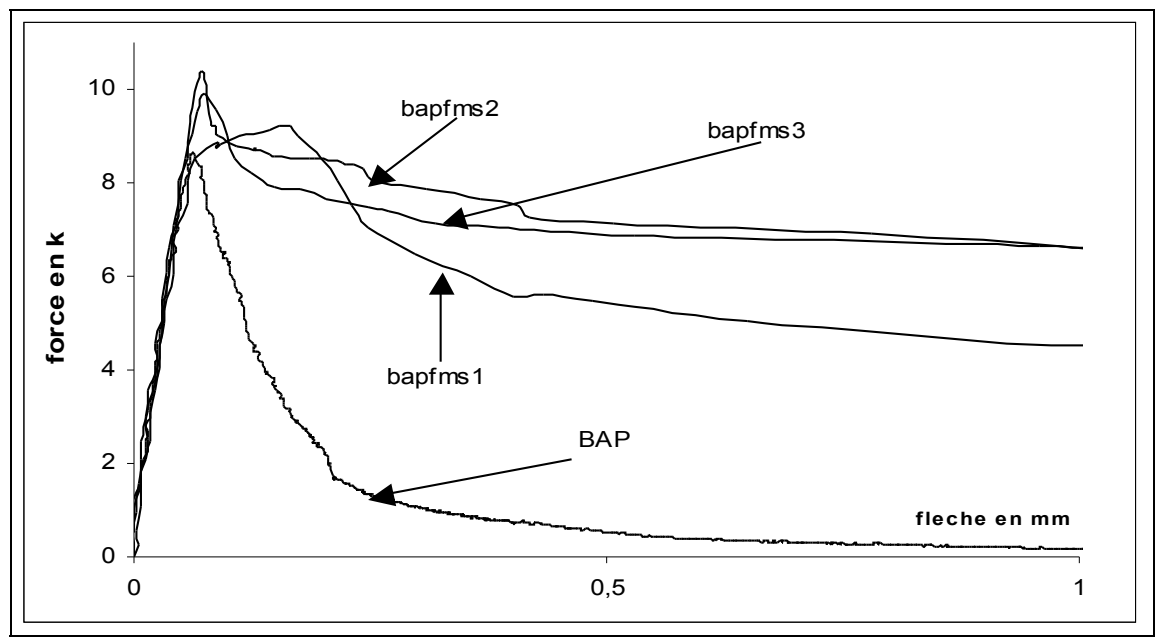

Figure 3 : Courbes effort-flèche : comparaison entre bapfmsl, bapfms2 et bapfms3 pour des flèches jusqu'à $1 \mathrm{~mm}$

\section{Cas des flèches mesurées jusqu'à $5,00 \mathrm{~mm}$ :}

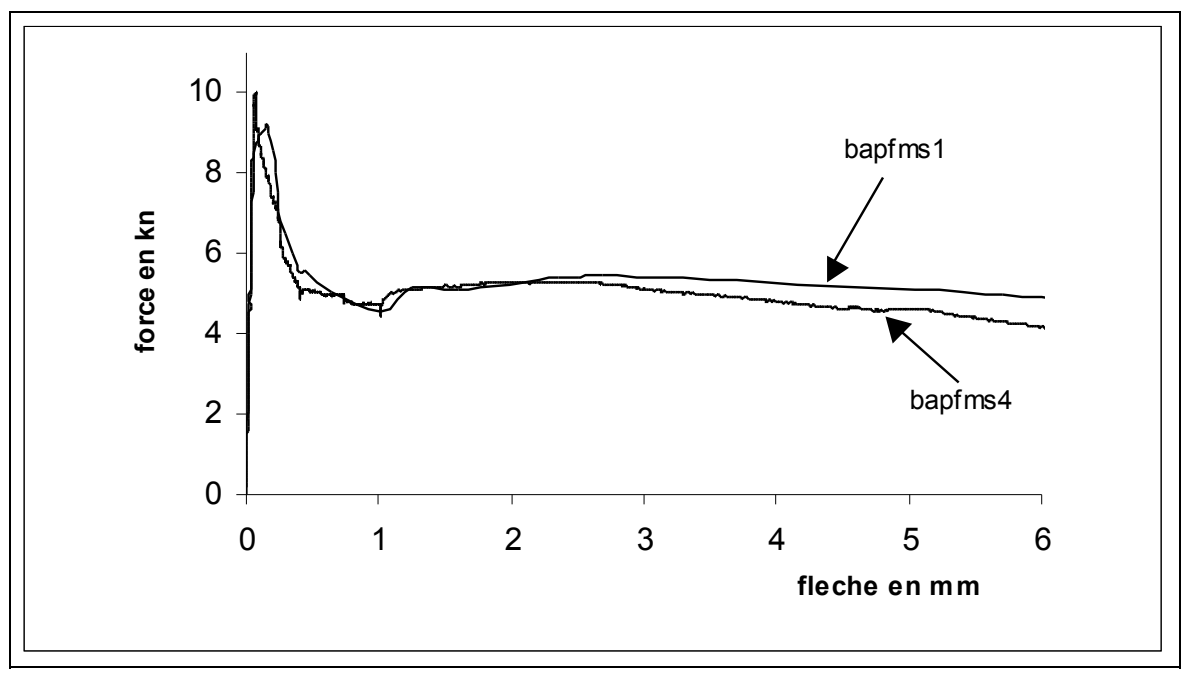

Figure 4 : Courbes effort-flèche : comparaison entre bapfmsl et bapfms4

Nous remarquons clairement que la capacité portante du BAPFMS4 est nettement supérieure malgré le dosage inférieur (moitié) comme le montre les courbes de la Figure 4. Ces résultats montrent bien l'intérêt des fibres courtes. Dans ce cas une teneur de $0,5 \%$ en fibres courtes a la même efficacité que l'utilisation de plus de $1 \%$ de fibres plus longues et ce pour une même composition de béton. 


\section{3-3. Approche énergétique}

Tableau 4: Energie totale consommée et indice de ténacité

\begin{tabular}{|c|c|c|c|c|c|}
\hline $\begin{array}{c}\text { Type de } \\
\text { béton }\end{array}$ & \multirow{2}{*}{$\sigma_{c}^{\prime}$ en Mpa } & $\begin{array}{c}\text { Energie en } \\
\text { Kn.mm }\end{array}$ & \multicolumn{3}{|c|}{ Indices de ténacité } \\
\cline { 3 - 6 } & & $\mathrm{E}_{5 \mathrm{~mm}}$ & $\mathrm{I}_{5}$ & $\mathrm{I}_{10}$ & $\mathrm{I}_{30}$ \\
\hline BAP & 36,03 & 2,30 & 3,06 & 3,85 & 4,55 \\
\hline BAPFMS1 & 38,50 & 30,72 & 3,20 & 4,65 & 8,31 \\
\hline BAPFMS2 & 38,36 & 38,12 & 3,33 & 6,20 & 15,70 \\
\hline BAPFMS3 & 41,40 & 39,04 & 3,26 & 10,74 & 18,80 \\
\hline BAPFMS4 & 36,90 & 29,07 & 3,43 & 5,90 & 10,87 \\
\hline BAPFMS5 & 36,50 & 22,80 & 3,52 & 5,54 & 10,15 \\
\hline
\end{tabular}

Pour chaque composition l'énergie a été calculée jusqu'à une flèche de $5 \mathrm{~mm}\left(\mathrm{E}_{5 \mathrm{~mm}}\right)$. Cette énergie est un indicatif de la ténacité du mélange, particulièrement dans le domaine post-fissuration [7]. Plus elle est grande plus le composite cimentaire est tenace. Les résultats sont présentés dans le Tableau 4 et traduits sous la forme de graphe barres sur la Figure 5.

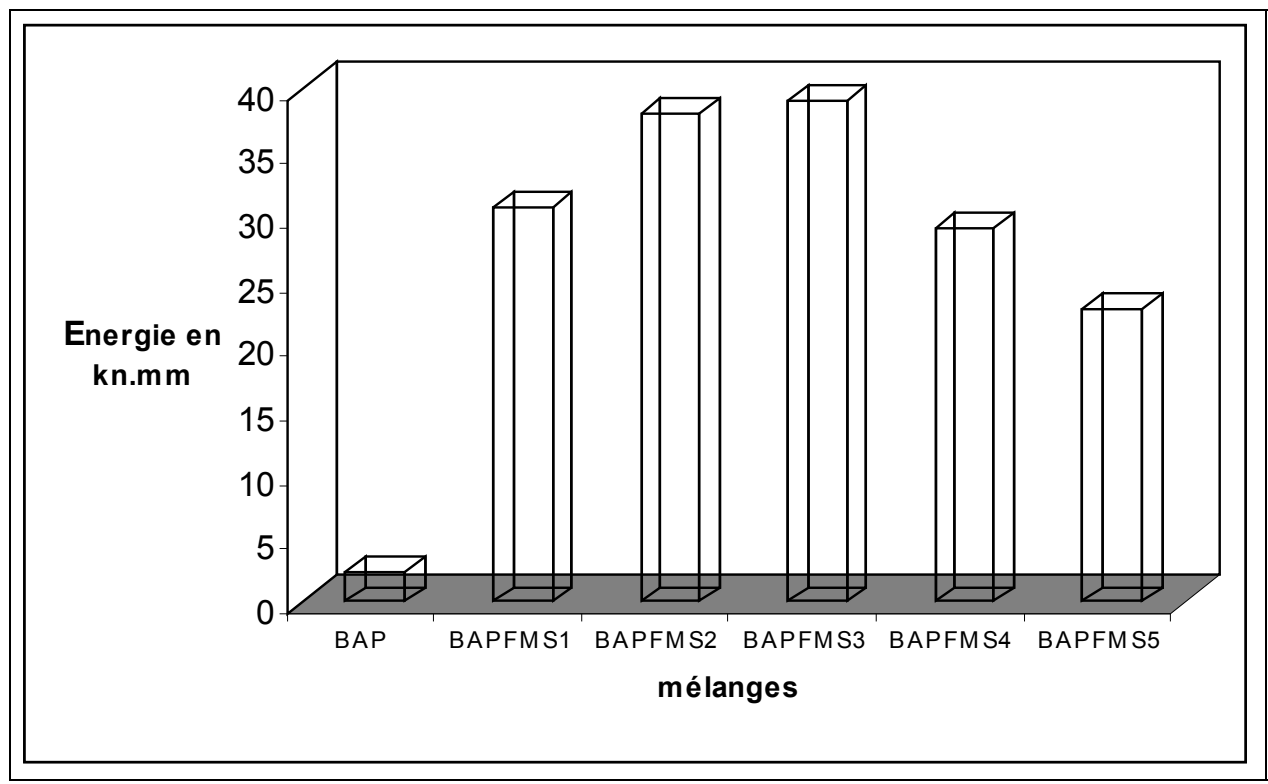

Figure 5 : Variation des énergies de rupture pour les différents mélanges 
Nous remarquons tout d'abord l'importance des fibres pour changer les conditions de ténacité des bétons auto-plaçants bi-fibrés. La valeur d'énergie la plus élevée est enregistrée pour BAPFMS3 qui contient exactement le même nombre de fibres métalliques que BAPFMSI mais de dimensions différentes (celles de BAPFMS3 sont plus courtes).

Les indices de ténacité au cours du chargement ont été également évalués pour chaque type de bétons et les résultats sont insérés dans le Tableav 4.

Pour chaque mélange, les énergies dissipées jusqu'à la première fissure ont été calculées. On remarque que la ténacité augmente avec tous les mélanges hybrides relativement au BAP sans fibre. Cependant le BAPFMSI donne les indices de ténacité les plus faibles relativement aux autres mélanges hybrides, mais une valeur d'énergie de rupture intermédiaire par rapport aux autres mélanges et ceci est probablement due aux longueurs de fibres métalliques (élancements). Les fibres courtes ce sont avérées le type le plus adapté dans de tels cas. Ces fibres métalliques courtes sont mélangées aléatoirement dans la matrice cimenteuse avec une ouvrabilité et une mise en œuvre remarquable qui ont permis une meilleure distribution uniforme [8]. La synergie (en terme d'indice de ténacité) entre les différents mélanges a été analysée et les meilleures performances sont obtenues avec les mélanges hybrides de fibres courtes, comme le montre d'ailleurs les valeurs du Tableau 4.

\section{Conclusion}

En soudant les macro-fissures et en réduisant leurs ouvertures, les fibres affectent évidemment la réponse du composite aussi bien au niveau du pic (capacité portante), qu'au niveau du palier post-pic (palier de ductilité). Les résultats obtenus confirment l'intérêt de la substitution des fibres longues par des fibres courtes tout en améliorant les propriétés rhéologiques et mécaniques. Les fibres de fonte amorphe assurent un retardement de l'apparition de la première fissure et une efficacité dans la restriction et ceci grâce à leur bonne adhérence et leur module d'élasticité très élevé. Les fibres en polypropylène glissantes procurent une réserve de sécurité importante pour le composite fibré en lui conférant un palier ductile, qui est d'un intérêt vital pour les BAP. Plusieurs constats peuvent être tirés de cette étude expérimentale :

En ce qui concerne les BAP bi-fibrés, on a pu, en fonction de la géométrie et de la nature des fibres employées, formuler un béton répondant aux critères rhéologique fixés.

A dosage égal, les fibres métalliques courtes ont donné une synergie (indice de ténacité) et énergie totale de déformation supérieures à celles des fibres longues. 
Les propriétés mécaniques, rhéologiques et synergiques sont plus favorables, avec les fibres métalliques relativement courtes.

D'autres essais, avec comme variable la longueur des fibres en polypropylène, sont actuellement en cours, et feront l'objet du prochain article dans la même revue.

\section{Remerciements}

Je tiens à remercier les responsables du laboratoire matériaux et durabilité des constructions (LMDC), INSA -UPS de Toulouse et notamment Mr A. Turatsinze, Maître de conférences et Mme G. Arliguie, Directrice du laboratoire pour m'avoir accueilli et aider ’̀ réaliser ce travail.

\section{Références}

[1] - MARCOVIC J. WALRAVEN ॥ Self-Compacting hybrid fiber Concrete-mix design, Workability and Mechanical Properties. II Third International Symposium on SelfCompacting Concrete, Reykjavik, (2003) pp. 763-775

[2] - P. ROSSI, II Les bétons de fibres métalliques II. Ed. Presse du LCPC, Paris (1998.)

[3] - G. PEIFFER ॥ Mechanical effectiveness ribbon - shapped fibers when used in reinforced concrete II, Centre de recherche de pont à mousson. (1991). Documentation du centre de recherche ponts à mousson

[4] - N. BANTHIA, R. GUPTA ॥ Hybrid fiber reinforced concrete (HyFRC): fiber synergy in high strength matrices 1 Materials and structures / Matériaux et Constructions, Vol.37 (December 2004), pp 707-716

[5] - A. KAWAMATA, H. MIHASHI, H. FUKUYAMA ॥ Properties of hybrid fiber reinforced cement-based composites. II Journal of advanced concrete Technology, 1-3, Japan concrete institute , (2003) pp 283-290

[6] - ALCANTARA MARCO ॥Bétons autoplaçants et fibrages hybrides: composition, rhéologie et comportement mécanique " Thèse de doctorat présentée à l'LMDC INSA Toulouse (Septembre 2004), $\mathrm{N}^{\circ} 16$

[7] - Wu Yao, Jie Li, Keru Wu I Mechanical properties of hybrid fiber-reinforced concrete at low fiber volume fraction. II Cement and Concrete Research 33 (2003) pp 27-30

[8] - J. L. GRANJU, V. SABATIER, M. ALCANTARA ॥ Hybrid fiber reinforcement of ordinary or self-compacting concrete II, BEFIB (2004), 20-22 0ct, Varenne (Italie) 